\title{
Cold Plasma Sterilization of Open Wounds: Live Rat Model
}

\author{
Danil Dobrynin, ${ }^{1}$ Kimberly Wasko, ${ }^{2}$ Gary Friedman, ${ }^{1}$ Alexander Fridman, ${ }^{3}$ \& \\ Gregory Fridman ${ }^{4, *}$
}

${ }^{1}$ Dept. of Electrical and Computer Engineering, Drexel University, ${ }^{2}$ Surgery, Drexel University College of Medicine, ${ }^{3}$ Dept. of Mechanical Engineering and Mechanics, Drexel University, ${ }^{4}$ School of Biomedical Engineering, Drexel University

*Address all correspondence to: Dr. Gregory Fridman; Drexel University, 3141 Chestnut Street, Philadelphia, PA 19104; 215-895-0576 ph, 215-895-1633 fax, gregfridman@gmail.com

\begin{abstract}
Atmospheric pressure non-equilibrium (cold) plasmas are already known to be effective sterilization agents. This work further confirms the ability of cold plasma to inactivate pathogenic organisms in a live animal model. The rat wound model used here represents a surgical treatment situation whereby the wound is open and the bleeding is controlled. High concentration of Staphylococcus aureus is placed onto the wound area and allowed to incubate for four hours prior to cold plasma treatment. We show a 3-log reduction in pathogen load on the wound following a one minute treatment.
\end{abstract}

KEY WORDS: cold plasma, non-thermal plasma, dielectric barrier discharge, floating electrode, sterilization, animal model, rat, plasma medicine

\section{INTRODUCTION}

Of the vast number of plasma applications in industry, uses of plasmas in biology and medicine have recently gained significant interes $\mathrm{t}^{1}$. One of the most widely discussed potential applications is plasma treatment of wounds for the purpose of reducing microbial load and enhancing healing processes ${ }^{2-6}$. Two major types of plasma treatment have been discussed in connection with wound disinfection. One is indirect treatment using gas flow through plasma which brings mostly neutral active species in contact with living tissue. The other is direct application of non-thermal plasma to living tissues ${ }^{3}$. Indirect treatment permits to decouple plasma system design from constraints related to applying the treatment to living tissues. For example, it permits to employ thermal plasma for generation of active species including substantial quantities of $\mathrm{NO}^{7-10}$. Indirect plasma treatment of skin and wounds has been investigated over the last several years demonstrating a significant effect ${ }^{4}$. On the other hand, applying non-thermal plasma directly to living tissue makes it possible to employ charges and short living neutral species ${ }^{11,12}$. It has been demonstrated that bacterial inactivation on surfaces of agar and even skin can be achieved significantly faster with direct exposure to non-thermal plasma such as the Dielectric Barrier Discharge (DBD) 3 . No significant toxicity was observed during relatively low power and duration plasma exposure which is typically sufficient to achieve significant reduction in the bacterial load ${ }^{4,8,12,13}$. However, effects of direct DBD plasma treatment on bacterial inactivation in real wounds have not been reported so far. 
In this paper we report on a study where open wounds contaminated with bacteria are treated with Floating Electrode -DBD plasma directly. Rat is employed as the animal model. Although bleeding is stopped and most of the excess wound fluid is removed prior to plasma application, the wound remains moist. The results demonstrate that over $3-\log$ reduction (over 1000 times) of bacteria occur following only $1 \mathrm{~min}$ of DBD plasma treatment without any visible tissue damage.

\section{MATERIALS AND METHODS}

The particular form of cold plasma used in this manuscript was used by authors in the previous in-vitro sterilization and blood coagulation studies ${ }^{12}$ and in the in-vivo for intact skin toxicity and sterilization ${ }^{4}$. The wound model was developed specifically for this study to simulate an open wound.

\section{A. Floating electrode dielectric barrier discharge}

In this study we have used a floating electrode dielectric barrier discharge (FE-DBD) generated between an insulated high voltage electrode and the sample (FE) undergoing treatment. Half millimeter thick polished clear fused quartz (Technical Glass Products, Painesville, $\mathrm{OH}$ ), was used as an insulating dielectric barrier. The setup and high voltage electrode schematic is shown in Figure 1. The discharge was generated by applying high voltage pulses with the following characteristics: $20 \mathrm{kV}(\mathrm{p}-\mathrm{p}), 1.6 \mu$ s pulse duration, $1 \mathrm{kHz}$ frequency. The average power density for the active area of the high voltage electrode was kept at the level of approximately $0.5 \mathrm{~W} / \mathrm{cm}^{2}$, i.e. 0.74 Watt for $15 \mathrm{~mm}$ electrode diameter. The electrode was specifically designed to fit into the Polycarbonate holder as is shown in Figure 1 and a set of spacers of $0.5 \mathrm{~mm}$ thickness was provided to the operator to allow for controlled application of plasma for every wound.
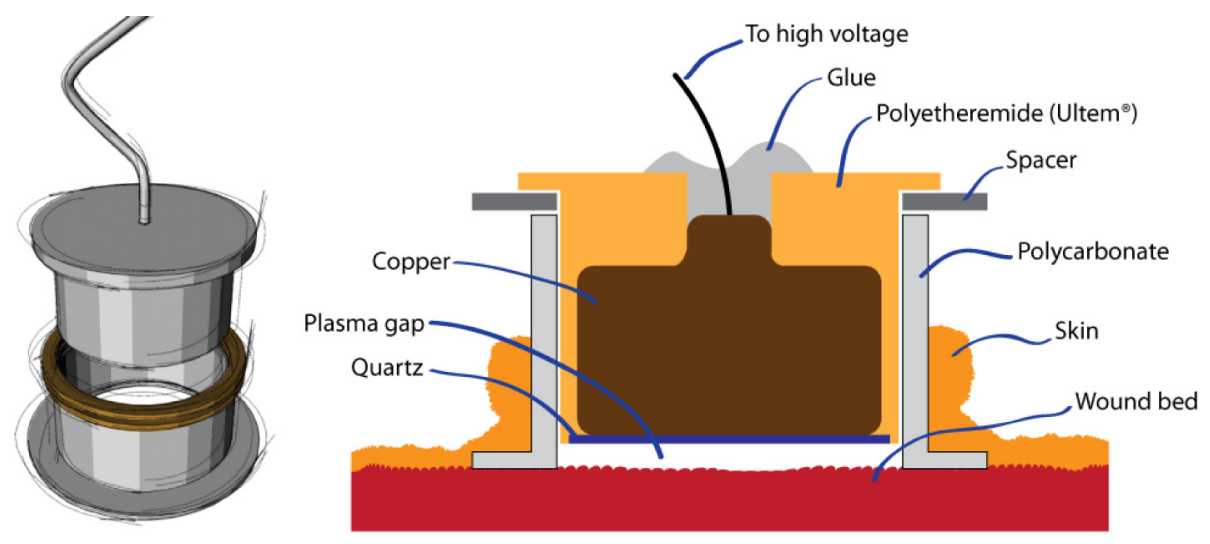

FIGURE 1. Schematic of the FE-DBD plasma electrode that fits into the plastic ring sutured to the wound. 
All procedures were performed in compliance with the animal welfare and protection act following the Drexel University's Institutional Animal Care and Use Committee (IACUC) approval.

\section{B. Animal model}

Thirty hairless Sprague-Dawley rats, weighing approximately $250 \mathrm{~g}$, arrived and acclimated for 3-5 days in the facility. Animals were transferred from the viviarium to the approved surgical suite. Animals were continuously anesthetized with inhalational gas anesthetic, Isoflurane (Vedco, St. Joseph, MO) at 2-3\% induction plus oxygen $2 \mathrm{~L} / \mathrm{min}$ then $1.5-2 \%$ maintenance administered via a face mask for the appropriate length of time and placed in the supine position. Animals received analgesia (Meloxicam, BoehringerIngelheim, Germany) at $1 \mathrm{mg} / \mathrm{kg}$, subcutaneous, Bupivicaine 25\% (Hospira, Lake Forest, IL) $0.5 \mathrm{ml} / \mathrm{site}$ intradermally and Normosol R (Baxter, Deerfield, IL) crystalloid parenteral fluid therapy at $10 \mathrm{ml} / \mathrm{kg}$ subcutaneous bolus at the onset of anesthesia but prior to surgery. The ventral surface of the rat was prepared in sterile surgical fashion: the surgical area was gently washed and sanitized with chlorhexidine scrub solution; the area underwent an initial contact scrub of 5 minutes, rinsed with normal saline solution and repeated second time; a preparatory solution of diluted chlorhexidine with isopropyl alcohol (1:8) was lightly sprayed onto the surgical area. Approximately $2 \mathrm{~cm}$ incisions along the right and left side of the thoracic region were inflicted and plastic rings were inserted into the subcutaneous layer of the skin (ring size was equivalent to the size of the plasma probe) and restricted the bacteria to the affected area only, Figure 2). The skin was sutured with 3-0 nylon around the ring in a continous suture pattern. Twenty $\mu \mathrm{l}$ drops of PBS solution containing $S$. aureus at concentration of $10^{5}$ or $10^{6} \mathrm{ml}^{-1}$ were applied to the wounds and covered and secured with sterile Tegaderm dressing (3M, St. Paul, MN). Animals were recovered from anesthesia in a pediatric warming incubator and bacteria incubated for four hours. Upon completion of bacterial incubation, animals were re-anesthetized, wounds were opened and treated with DBD or untreated controls. After treatment, wounds were swabbed using sterile technique and transferred into PBS for further dilution and plating and plated directly onto BHI agar. Bacteria inactivation efficiency by DBD plasma treatment was analyzed after 14 hours of incubation on BHI agar. Figure 2 shows the sequence of steps taken during the animal procedure.

All data is reported with $95 \%$ confidence interval $(\mathrm{p}<0.05)$ and the number of samples in each experiment is identified.

\section{RESULTS AND DISCUSSION}

The results of viable Staphylococcus aureus inactivation in living tissue of experimental wound using FE-DBD plasma for initial concentration of bacteria of $10^{5}$ or $10^{6}$ cells per $\mathrm{ml}$ of PBS are shown in Figure 3. We observed a 3-log reduction of bacteria after a one minute exposure to plasma, and the results are similar for the both initial concentrations of bacteria. 


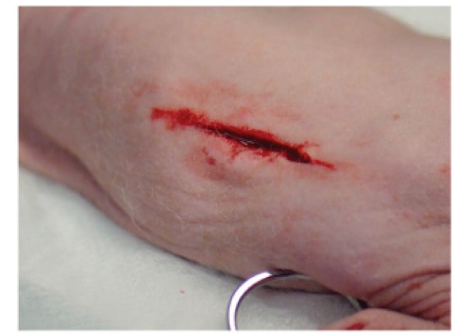

(a) $2 \mathrm{~cm}$ SQ incision

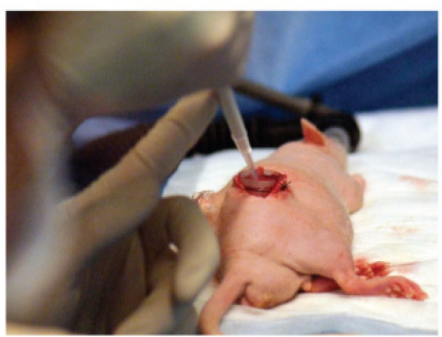

(d) Bacterial application

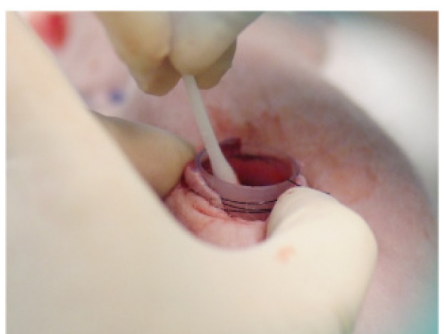

(g) Wound sample collection

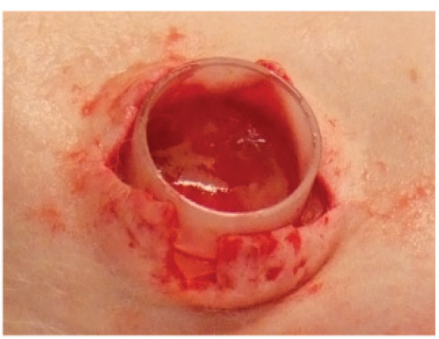

(b) Ring insertion

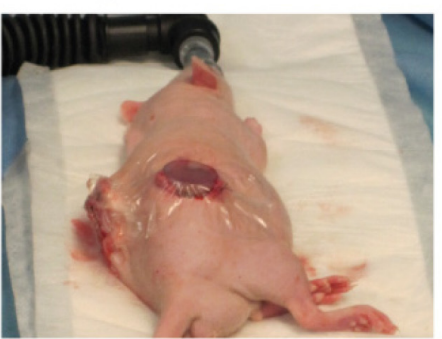

(e) Tegader $^{\circledast}$ dressing

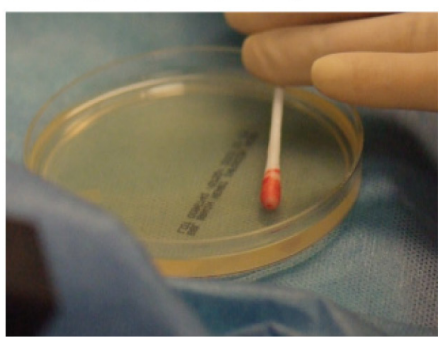

(h) Wound sample plating

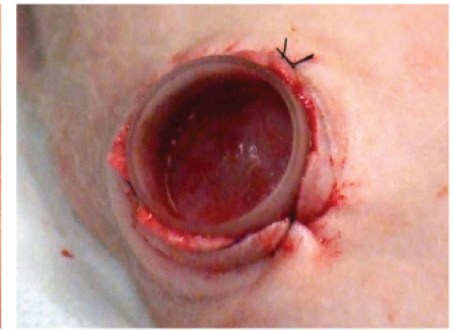

(c) Wound closure

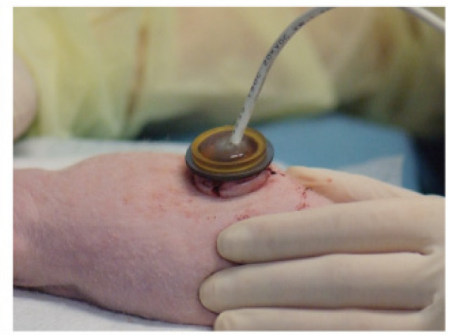

(f) Plasma treatment

FIGURE 2. Steps taken during the animal procedure: (a) a cut is made; (b) plastic ring is inserted into the skin and (c) sutured in place; (d) wound area is inoculated with known concentration of $\mathrm{S}$. aureus and (e) covered with sterile dressing; 4 hours following inoculation the sterile covering is removed and (f) wound area is treated with plasma; after the treatment $(\mathrm{g})$ the wound area is thoroughly swabbed and $(\mathrm{h})$ plated for bacterial counts.

Interestingly, the inactivation efficiency of plasma treatment in the wound is about an order of magnitude less than that for the agar surface ${ }^{4,12}$. Here we propose two possible explanations of this effect. First, it is necessary to mention that even under assumption of discharge uniformity (i.e. discharge was covering the whole surface of the electrode, which in present setup was impossible to control), direct plasma exposure has provided only at $\sim 75 \%$ of the wound surface due to presence of dielectric enclosure around central copper. Therefore, it is possible that the remaining $\sim 25 \%$ of bacteria, which were relatively uniformly distributed over the wound, were treated indirectly. Indirect plasma exposure, i.e. when there is no direct exposure of the treated bacteria to charged species, was previously shown to be about an order of magnitude less effective 


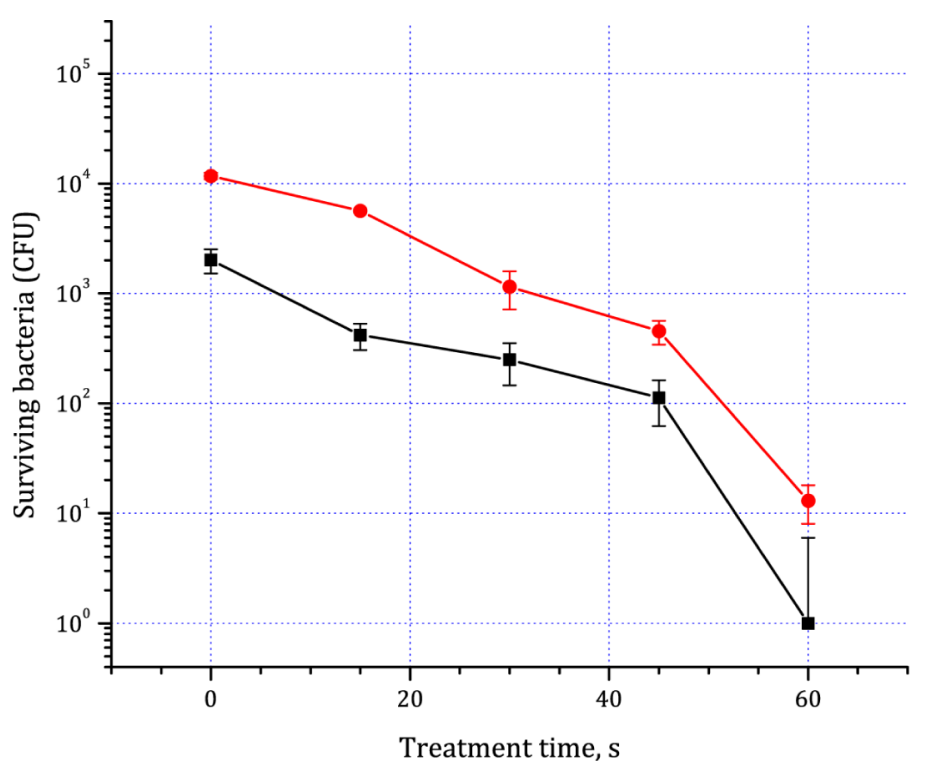

FIGURE 3. Results of wound sterilization with 103 and 104 starting bacterial load.

in bacteria inactivation on agar than direct treatment ${ }^{3}$. The second hypothesis is related to the presence of wound liquids - compared to almost dry, covered with a minute amount of water, "moist" surface of agar. Again, as we have previously shown, liquid water plays as a "protective" layer, preventing direct delivery of charges to the bacteria surface, and significantly dilutes the inactivation effect of active neutral particles ${ }^{3,11}$. At the same time, this wound liquid, which contains high amount of organic molecules, and wound itself may also react with plasma produced reactive species, resulting in decreased activity of plasma treated medium.

\section{CONCLUSION}

While we have previously shown effective inactivation of pathogenic organisms in many regimes of plasma and on various surfaces (see ${ }^{4,11}$ for examples) we have not previously addressed sterilization of living open wound tissue such as would be present during surgery. In this manuscript we show that sterilization is possible and is quite effective of an open wound area of bacteria that was allowed to "settle" in the wound for 4 hours. While the treatment appears to not have any adverse or toxic effect on the wound tissue (as was previously the case with intact $\operatorname{skin}^{12}$ ) an open question remains of the effect of plasma on the wound itself and on the wound healing rate. Since plasma was previously shown in-vitro to generate reactive oxygen species (ROS) which were shown to promote cell proliferation ${ }^{13}$ and in this work was confirmed in-vivo to produce bacterial inactivation similar to in-vitro studies we may assume that wound healing time will be reduced following the treatment. This, however, remains to be verified and is the subject of our follow-on studies. 


\section{REFERENCES}

1. Council, P.C.P.S.C.N.R., Plasma Science: Advancing Knowledge in the National Interest. 2007: The National Academies Press.

2. Martines, E., M. Zuin, R. Cavazzana, E. Gazza, G. Serianni, S. Spagnolo, M. Spolaore, A. Leonardi, V. Deligianni, P. Brun, M. Aragona, and I. Castagliuolo, A novel plasma source for sterilization of living tissues. New Journal of Physics, 2009. 11.

3. Fridman, G., A.D. Brooks, M. Balasubramanian, A. Fridman, A. Gutsol, V.N. Vasilets, H. Ayan, and G. Friedman, Comparison of Direct and Indirect Effects of Non-Thermal Atmospheric Pressure Plasma on Bacteria. Plasma Processes and Polymers, 2007. 4: p. 370-375.

4. Fridman, G., G. Friedman, A. Gutsol, A.B. Shekhter, V.N. Vasilets, and A. Fridman, Applied Plasma Medicine. Plasma Processes and Polymers, 2008. 5(6): p. 503-533.

5. Weltmann, K.D., E. Kindel, T. von Woedtke, M. Hahnel, M. Stieber, and R. Brandenburg, Atmospheric-pressure plasma sources: Prospective tools for plasma medicine. Pure and Applied Chemistry, 2010. 82(6): p. 1223-1237.

6. Vandamme, M., E. Robert, S. Pesnel, E. Barbosa, S. Dozias, J. Sobilo, S. Lerondel, A. Le Pape, and J.M. Pouvesle, Antitumor Effect of Plasma Treatment on U87 Glioma Xenografts: Preliminary Results. Plasma Processes and Polymers, 2010. 7(3-4): p. 264-273.

7. Isbary, G., G. Morfill, H.U. Schmidt, M. Georgi, K. Ramrath, J. Heinlin, S. Karrer, M. Landthaler, T. Shimizu, B. Steffes, W. Bunk, R. Monetti, J.L. Zimmermann, R. Pompl, and W. Stolz, A first prospective randomized controlled trial to decrease bacterial load using cold atmospheric argon plasma on chronic wounds in patients. British Journal of Dermatology, 2010. 163(1): p. 78-82.

8. Kong, M., G. Kroesen, G. Morfill, T. Nosenko, T. Shimizu, J. Dijk, and J. Zimmermann, Plasma medicine: an introductory review. New Journal of Physics, 2009. 11: p. 115012.

9. Morfill, G.E., T. Shimizu, B. Steffes, and H.U. Schmidt, Nosocomial infections-a new approach towards preventive medicine using plasmas. New Journal of Physics, 2009. 11.

10. Gostev, V. and D. Dobrynin. Medical microplasmatron. in 3rd International Workshop on Microplasmas (IWM-2006). 2006. Greifswald, Germany.

11. Dobrynin, D., G. Fridman, G. Friedman, and A. Fridman, Physical and biological mechanisms of direct plasma interaction with living tissue. New Journal of Physics, 2009(11): p. 115020.

12. Fridman, G., M. Peddinghaus, H. Ayan, A. Fridman, M. Balasubramanian, A. Gutsol, A. Brooks, and G. Friedman, Blood coagulation and living tissue sterilization by floating-electrode dielectric barrier discharge in air. Plasma Chemistry and Plasma Processing, 2006. 26(4): p. 425-442.

13. Kalghatgi, S., G. Friedman, A. Fridman, and A. Clyne, Endothelial Cell Proliferation is Enhanced by Low Dose Non-Thermal Plasma Through Fibroblast Growth Factor-2 Release. Annals of Biomedical Engineering, 2010. 38(3): p. 748-757. 